\title{
Drug Delivery Microdevice
}

National Cancer Institute

\section{Source}

National Cancer Institute. Drug Delivery Microdevice. NCI Thesaurus. Code C162994.

A drug delivery system integ rated within a single micro/nanofluidic chip, designed to provide even distribution to the whole body through blood circulation. 